\title{
Nuclear shapes
}

Renée Lucas

7 he naive picture of spherical nuclei is $I$ far from reflecting the reality of nuclear structure. Indeed a variety of different shapes have been observed and/or predicted that depend on the neutron to proton ratio and on the conditions of excitation energy or spin of nuclei. Thanks to the impressive technical developments of $\gamma$-ray detector arrays, the interest of nuclear physicists in nuclear structure related to the study of nuclear shapes, in particular at high angular momenta, has become more and more important.

Indeed, very few nuclei have a spherical shape in their ground state and a variety of shapes can be observed, often in the same nucleus. The deformed nuclei can be schematically subdivided into prolate, oblate and triaxial deformed nuclei depending on the relative axis values of the ellipsoid. Other shapes can also be encountered (fig.1). The low lying part of the energy spectrum of spherical nuclei can be interpreted in terms of surface vibrations. Magic or semi-magic nuclei (see box 1) have very few excited states at low excitation energy, their low transition probabilities indicating a low collectivity of these states and the dominance of single particle degrees of freedom. In contrast, the energy spectrum of a deformed nucleus has excited levels at energies which obey the typical relation of the angular momentum I for a rigid quantum mechanical rotor: $\mathrm{E} \sim \mathrm{I}(\mathrm{I}+1)$. The measured quadrupole moments $(\mathrm{I}(\mathrm{I}+1) / 2 \hat{\mathrm{J}}$ where $\hat{\mathrm{J}}$ is the dynamical moment of inertia) for these nuclei characterise their deforma- tion. As nuclear rotation also modifies the microscopic structure of the nucleus, the shapes favoured by a particular nucleus will change with increasing spin. Of course, many nuclei have a transitional character involving both vibrational and rotational features.

Near closed shells spherical shapes prevail, while between closed shells the large number of valence nucleons in orbits with large single particle angular momentum leads to nuclei with large deformations. It is also possible to observe both a deformed and a spherical shape in the same nucleus at different excitation energies and spins. Fig 2 gives an overview of the different shapes encountered or predicted presented over the chart of nuclei. One has, however, to remember that theoretical predictions and recent measurements indicate that very far from stability, for large $N / Z$ ratios (number of neutrons over number of protons), there might be a quenching of shells changing the representations on the chart of deformed nuclei.

Nuclear structure research with exotic as well as stable nuclear beams is in an exciting and evolving challenge. By choosing a stable or exotic beam one can reach nuclei far from the stability line and at different excitation energies. This indicates the importance of access to different and complementary facilities. In Europe such facilities are GSI (Darmstadt Germany), JYFL (Jyväskilä Finland), Vivitron (Strasbourg France) for the stable beams, or GANIL (Caen France) and CRC (Louvain la Neuve Belgium) for radioactive beams. One of the main tools used to determine nuclear properties is $\gamma$-ray spectroscopy and the impressive improvements made in detector instruments during the last decade have lead to an extensive range of new results. New $\gamma$-ray detectors, such as EUROBALL and GAMMASPHERE, are being used either alone or in association with other instruments such as detectors for conversion electron, charged particle or fission fragments, or recoil spectrometers, or velocity filters.

To show how lively and diversified this research domain is today, some examples of nuclear shapes encountered in the different regions of the chart of nuclei and for different experimental conditions are given below. This cannot be an exhaustive presentation of the recent results and this article should be considered only as an attempt to give a flavour of the field.

\section{Deformed ground state of nuclei}

\section{Light nuclei}

In light nuclei it is possible to explore an isotopic chain from the most neutron rich elements to the proton drip line. By Coulomb excitation one has gained access to nuclear parameters such as the energy of the first excited state and its excitation probability. For example, the large excitation probability for the first excited $2^{+}$state found for ${ }_{16}^{44} \mathrm{~S}$ indicates that this nucleus has not the spherical shape expected from the $\mathrm{N}=28$ magic number of neutrons [ref. 1]. Neutron-rich magnesium isotopes have been extensively investigated from both theoretical and experimental points of view. According to microscopic calculations their structure fluctuates very rapidly from one isotope to the next and the ${ }_{12}^{32} \mathrm{Mg}$ ground state is deformed, in spite of the $N=20$ shell closure. Indeed the low energy of the first excited $2^{+}$state $\lambda=0$ Sphere

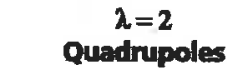

$\lambda=3$ Octupoles $\lambda=4$ Hexadecapoles

OBLATE

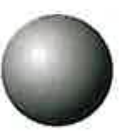

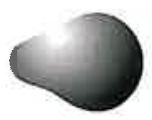
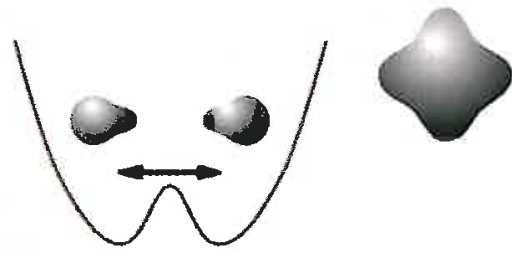

PROLATE
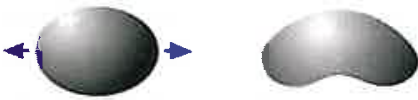

Fig. 1: Currently observed nuclear shapes. The different shapes can be parametrized by spherical harmonic functions, where $\lambda$ characterises the different orders of the corresponding distributions. and the large transition prob-
ability are in agreement with such a deformed shape. Experiments have been recently carried out to determine more precisely the deformation parameters.

\section{Heavy nuclei}

The rotational character of the ground state bands of deformed nuclei have been intensively studied. As far back as 1981, a Coulomb excitation experiment of ${ }_{92}^{238} \mathrm{U}$ was made at GSI (Darmstadt) [ref. 4]. Coulomb excitation was pre- 
ferred to a (heavy ion, $\mathrm{xn}$ ) reaction, the former reaction being favoured by the large intrinsic quadrupole moment of ${ }_{92}^{238} \mathrm{U}$ and offering a good possibility to study high spin effects. The rotational band of ${ }_{92}^{238} \mathrm{U}$ was observed up to spin $32 \hbar$ in accordance with a single particle picture and collective approach. The transition energies in the ground state band show a regular increase with increasing spin up to the higher spin. The measured transition probabilities correspond to an axially deformed nucleus rotating about an axis perpendicular to its symmetry axis and having a constant electric quadrupole moment.

\section{Very heavy nuclei}

The structure of the heaviest elements still remains a challenge to experimental and theoretical studies. The theoretical predictions of sizeable deformations can only be confronted in a few experiments. The formation mechanism and the shape of the fission barriers are not completely understood. It is important to study the underlying shell structure of nuclear shapes for the search of even heavier nuclei. Recently the coupling of recoil separators with efficient $\gamma$-arrays (FMA (Fragment Mass Analyser) + GAMMASPHERE at Argonne and RITU (Recoil Ion Transport Unit) + JUROSPHERE at Jyväskilä) has enabled the study of decay schemes with production cross sections below $1 \mu \mathrm{b}$ in the study of ${ }_{102}^{254} \mathrm{No}$ and ${ }_{102}^{252} \mathrm{No}$ [ref. 5 ]. The measured rotational bands extend up to spin $16^{+}$in ${ }_{102}^{25} \mathrm{No}$ and to $18^{+}$for ${ }_{102}^{252} \mathrm{No}$. The first sets of gamma spectroscopic data on transfermium nuclei have proven that, in accordance with theoretical predictions, ${ }_{102}^{254} \mathrm{No}$ and ${ }_{102}^{252} \mathrm{No}$ are deformed nuclei with an axis ratio of about 1:1.3. By comparing the population of the ground state bands in ${ }_{102}^{254} \mathrm{No}$ and ${ }_{102}^{252} \mathrm{No}$, the influence of the neutron shell closure can be studied. The study of nuclear structure of nuclei with $\mathrm{Z}>100$ is in full development.

\section{Shape transitions and shape coexistence}

In an isotopic chain, shape transitions of the ground states can occur with the variation of neutron numbers. They can be identified either from the large differences in the energies of the first excited states (for instance the energy of the $2^{+}{ }_{1}$ state suddenly drops from $1223 \mathrm{keV}$ in ${ }_{40}^{98} \mathrm{Zr}$ to $270 \mathrm{keV}$ in ${ }_{40}^{100} \mathrm{Zr}$ ), or from the variation of the measured mean square radii (such as in $\mathrm{Hg}, \mathrm{Au}$ or Pt isotopes).

Ambiguous results can be clarified by observing the shape evolution as a function of the neutron number along a com-

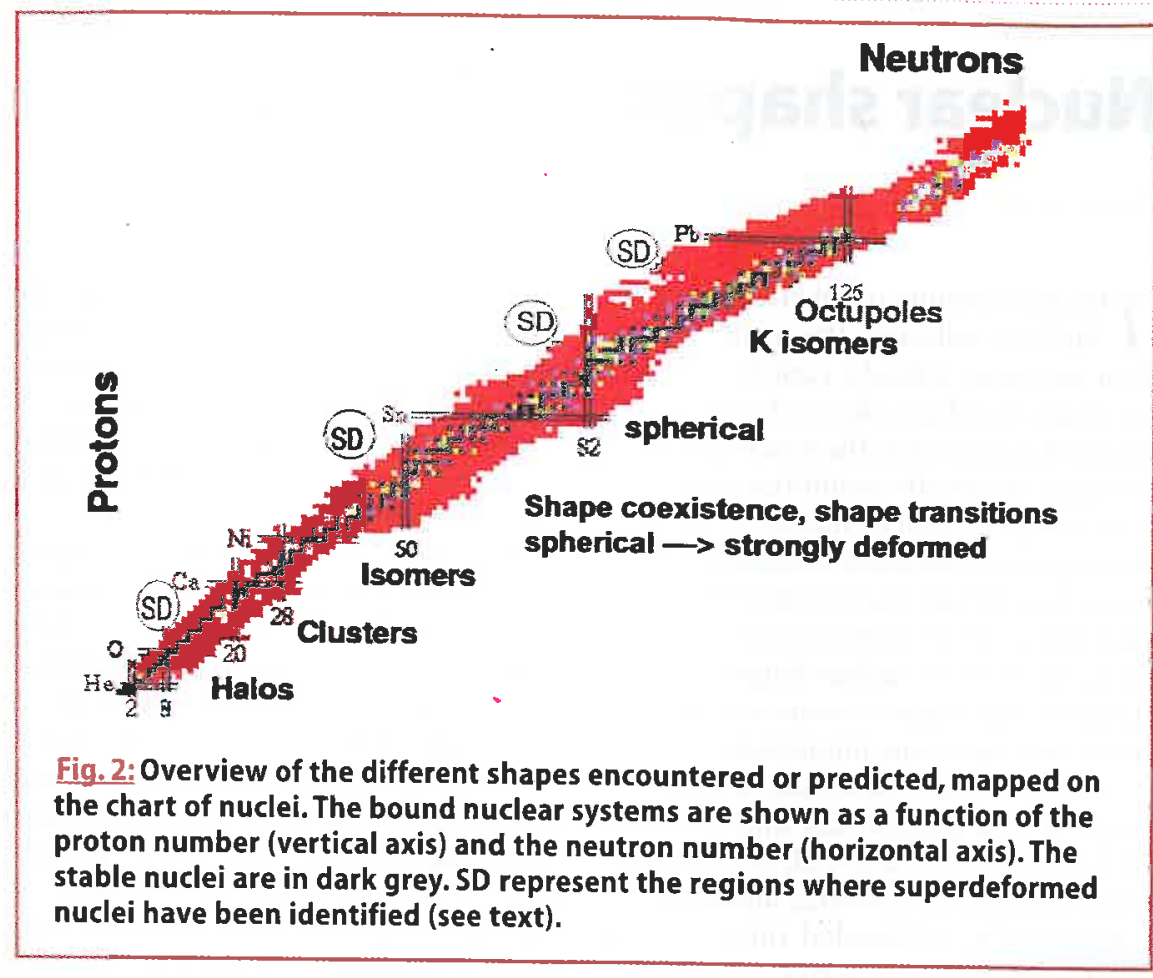

plete chain of isotopes. For example a fusion-fission reaction has been used to study neutron-rich palladium nuclei (which cannot be reached in any other manner until the advent of new radioactive beams), thereby completing the systematics of nuclear structure studies from ${ }_{46}^{106} \mathrm{Pd}$ to ${ }_{46}^{118} \mathrm{Pd}$ [ref. 6].

Shape coexistence phenomenon (the existence of two stable shapes at the same excitation energy) can inform us upon the interplay between single particle and collective degrees of freedom. The nuclei in the mass region around $\mathrm{A} \sim 70-80$, especially those close to the $\mathrm{N}=\mathrm{Z}$ line, are predicted to exhibit a wide variety of shapes including large prolate and oblate deformations, triaxial and spherical shapes. Several $0^{+}$states are expected at low excitation energy. They can be isomeric states (states with strongly inhibited electromagnetic decay modes) and decay to the ground state by means of electric monopole transitions. Evidence for such E0 transitions, which are directly linked to a change in the mean square radius of the nucleus, has been found in ${ }_{34}^{72} \mathrm{Se},{ }_{34}^{74} \mathrm{Se}$, and recently in ${ }_{36}^{74} \mathrm{Kr}$. The shape of the yrast band ${ }^{1}$ appears to change from prolate for ${ }_{36}^{76,74} \mathrm{Kr}$ to oblate for ${ }_{36}^{80} \mathrm{Kr}$. Coulomb excitation experiments might give a full characterisation of the low lying collective states in the intermediate nucleus ${ }_{36}^{78} \mathrm{Kr}$.

Another example is given by the neutron deficient lead isotopes just below the doubly-magic ${ }^{208} \mathrm{~Pb}$ which reveal many different aspects of shape coexistence at both low and high spins. Recent evidence for such a coexistence has come from the observation of excited $0^{+}$states and rotational bands in ${ }^{184-190} \mathrm{~Pb}$. These experiments were made in Canberra [ref. 7] and in Finland [ref. 8]. The existence of two excited low-lying $0^{+}$states have been confirmed in ${ }_{82}^{208} \mathrm{~Pb}$ that has been interpreted as evidence for a triple shape coexistence. The microscopic structure of the coexisting states in the lead isotopes and the development of shape coexistence have yet to be studied thoroughly by the complete repertoire of available spectroscopy.

\section{K Isomers}

The study of isomeric states provides direct information on microscopic nuclear structure effects. In deformed axially symmetric nuclei, isomers occur when there is a secondary minimum for a certain value of $\mathrm{K}$, the projection of the total nuclear spin along the symmetry axis of the nucleus (fig 3 ). Trapped in this pocket, it is difficult for them to change their spin orientation relative to an axis of symmetry. These isomers are known as $\mathrm{K}$ traps. High $\mathrm{K}$ isomers are known in the $\mathrm{A} \sim 180$ mass region for neutron deficient isotopes of $W$, Hf and $O s$ which have a prolate shape. The occurrence of $\mathrm{K}$ isomers in neutron rich nuclei have been predicted and tested in many cases [ref. 9]. Recent experiments carried out at GSI (Darmstadt) [ref. 10] using projectile fragmentation of ${ }_{82}^{208} \mathrm{~Pb}$ at relativistic energy (here $1 \mathrm{GeV} / \mathrm{A}$ ) has led to the population of excited states in ${ }_{76}^{190} \mathrm{~W}$. 
momentum in lighter nuclei. The success of these models was illustrated by the discovery in 1986 of the first superdeformed band in ${ }_{66}^{152} \mathrm{Dy}$. The characteristic picket fence gamma-ray pattern of a rotational band was observed for spins as high as $60 \%$ and the measurement of the quadrupole moment indicates a deformation with an axis ratio of $2: 1$. Since then, using fusion evaporation reactions with stable beams, superdeformed nuclei have been discovered in distinct regions of the periodic table with masses around $60,90,130,150$, 190 and 240 and with axis ratios from 1.3 to 2 .

Although strong progress have been made in research on superdeformation many questions remain open [ref. 12]. The major challenge is to determine the excitation energy and spins of superdeformed levels. In the different mass regions, the linking transitions between SD and normal deformed states have only been established for some nuclei such as, for example, ${ }_{30}^{60} \mathrm{Zn},{ }_{64}^{149} \mathrm{Gd},{ }_{80}^{194} \mathrm{Hg}$ and ${ }_{82}^{194} \mathrm{~Pb}$. Therefore, almost all superdeformed bands discovered so far are "floating bands" as the transitions connecting a SD band with low lying states are not known. This is probably due to the strong fragmentation of the decay into many paths, thus requiring high-fold coincidences for their observation. The new generation of $\gamma$-arrays associated with auxiliary detectors such as electron conversion spectrometers will help to find these links as E0 transitions may compete with $\gamma$-ray transitions. If the deformation of the states is known, the E0 decay strength may give information on the mixing of the wave functions.

The extreme regularity of SD bands has allowed the study of deviations from expected behaviour in a very accurate way. Hence, the phenomenon of "identical bands" (neighbouring nuclei rotating with exactly the same rotational frequency, within about two parts in a thousand) was first noted in SD bands, but has now been found to be a common feature of rotational bands in normally deformed nuclei. This phenomenon is not yet quantitatively understood and deserves other studies. Another surprising phenomenon is the observation of the oscillations of the moments of inertia, has been tentatively explained by the onset of new types of symmetry involving the $\mathrm{C} 4$ point group symmetry [ref .13].

\section{Perspectives}

The borders of the SD regions have yet to be established. Since all identified superdeformed bands are populated via fu-

\section{Box 2: Isomers}

Isomeric states, states with strongly inhibited electromagnetic decay modes, occur when there is a secondary energy minimum for some values of shape elongation, spin or spin projection on the symmetry axis. This leads to the three main configurations schematically presented in fig 3 . Shape isomers can decay by $\gamma$-ray emission or by fission if the fission barrier is low enough (fission isomers). The facility for spin isomers trapped in the energy pocket to decay by $\gamma$-ray towards lower energy states depends on the selection rules. For $K$ isomers, the orientation of the nuclear spin vector has also to be taken into account.

sion-evaporation reactions, only neutron deficient nuclei are reached. Thus the discovery of superdeformed bands in neutron rich regions is still a challenge. It is also expected that octupole correlations will play an important role in the structure of superdeformed states. Moreover, actinide nuclei open a unique opportunity to investigate also the structure of SD nuclei at low angular momentum. Tentative experiments to populate these states by transfer reactions have as yet proved unfruitful. The continuous improvement of detector systems should, it is hoped, allow one to reach this objective.

A third well with hyperdeformed shape (axis ratio 3:1) has been identified in thorium isotopes from resonant states in the $(d, p)$ reaction [ref. 14]. Nevertheless no hyperdeformed band has been measured yet in $\gamma$ spectroscopy, although several theoretical calculations predict that states with very elongated shapes become yrast for spins I 70h in some nuclei, such as ${ }_{64}^{146} \mathrm{Gd}$ or ${ }_{72}^{168} \mathrm{Hf}$ [ref. 15]. At this very high spin value the stabilisation of secondary minima in the potential well may compete with the vanishing of the fission barrier making observation of such a very deformed state very difficult. This will be one of the main challenges in the next coming years.

The various examples given show the variety of possibilities offered by the choice of beam (stable or radioactive) and the dependence of the outcome on the excitation energy attained, on channel-entrance effects (direct or inverse kinematics) and on the different types of reactions studied (fusion-evaporation, transfer, fission and coulomb excitation). More and more sophisticated gamma arrays and auxiliary instruments (neutron, charged particle and fission fragment detectors) have to be used to observe and chart the many facets of nuclear shapes. The conjunction of all the usable means will allow the thorough study of this field of nuclear structure to be extended for nuclei further and further from stability.

\section{References}

[1] T. Glasmacher et al Phys. Lett. B 395 (1997) 163

[2] B. Jonson and K. Riisager Phil Trans (1998)

[3] A.H. Wuosmaa et al Ann. Rev. Nucl. Part. Sci 45 (1995) 189

[4] E. Grosse Phys. Scripta Vol 24, 337 (1981)

[5] M. Leino Eur. Phys. J. A 6, 63 (1999)

[6] M. Houry et al Eur. Phys. J. A 6, 43 (1999)

[7] G.D. Dracoulis et al Phys. Rev. C60 (1999) 014303 and references therein

[8] J.F.C. Cocks et al Eur. Phys. J. A 3, 17 (1998)

[9] P. Walker and G. Dracoulis Nature 399 (1999) 35

[10] Z. Podolyak et al submitted to Phys. Rev. C

[11] I. Ahmad and P. Butler Ann. Rev. Nucl. Part. Sci (1993) 4771

[12] P.J. Nolan and P.J. Twin Ann. Rev. Nucl. Part. Sci. (1988) 38533

[13] B. Haas Europhys. News 26 (1995) 103 [14] J. Blons Nud. Phys. A502 (1989) 121c; P. Glässel et al Nucl. Phys. A256, 220 (1976)

[15] B. Herskind Workshop on gamma spectroscopy Strasbourg (1999)

\section{Further reading}

Clusters: A.H. Wuosmaa et al Ann. Rev. Nucl. Part. Sci (1995) 45189

Shape coexistence: J.L. Wood et al Physics Reports Vol 215 (3\&4) 101 (1992)

Octupoles: I. Ahmad and P. Butler Ann. Rev. Nucl. Part. Sci (1993) 4771

Halos: B. Jonson and K. Riisager Internal Report CTHSP 97-08 (1997)

$\mathrm{K}$ isomers: P. Walker and G. Dracoulis Nature 399 (1999) 35

Superdeformed shapes: P.J. Nolan and P.J. Twin Ann. Rev. Nucl. Part. Sci. (1998) 38 533

Hyperdeformed nuclei: J. Blons Nucl. Phys. A502 (1989) 121c and references therein

P. Glässel et al Nucl. Phys. A256, 220 (1976) and references therein 